\title{
Protect the player, protect the game: subconcussion and chronic traumatic encephalopathy.
}

\author{
Jong Hyun Lee ${ }^{1}$ and Keisuke Kawata $\mathrm{PhD}^{1,2}$ * \\ ${ }^{1}$ Department of Neuroscience, Lewis Katz School of Medicine at Temple University, Philadelphia, PA, USA \\ ${ }^{2}$ Department of Kinesiology, Indiana University, Bloomington, IN, USA
}

Accepted on September 12, 2016

Chronic traumatic encephalopathy (CTE), formerly known as "punch drunk" or dementia pugilistica, is a progressive neurodegenerative disease. Repetitive exposure to head insults may cause insidious effects in the brain parenchyma, including tau protein dissociation and abnormal phosphorylation, encompassing the cerebrovasculature without being cleared. Concomitantly, various psychosocial complications (e.g., headache, difficulty concentrating, depression, dementia) are notable in CTE.[1,2] However, because the bulk of evidence originates from post-mortem case reports, there is a substantial knowledge gap in causality among tau accumulation, head impact exposure, and behavioral alteration.

Previously, boxing had been the only domain in which CTE was observed and studied. However, recent case series suggests that abnormal tau deposits are also endemic in some of contact prone athletes (e.g., American-football, ice hockey, soccer) and military personals.[3,4] Post-mortem analysis revealed that pathological characteristics of CTE include deterioration of brain structures such as hippocampus, medial temporal region, and frontal lobes.[5] At advanced stages, excessive cerebrospinal fluid expanding the crevice between the two leaflets of the septum pellucidum can be observed[3].

While antemortem diagnosis of CTE remains challenging, the disease can be progressive and predictive once the early symptoms, due to brain parenchymal damage, are clinically detectable and significant. According to the pioneer studies by Dr. McKee and her colleagues[1], CTE can be classified into four stages: stage 1 exhibits chronic headaches and difficulty concentrating; stage 2 involves inability to control emotions and temper, prone to violence, short-term memory loss, anxiety, and depression; stage 3 shows signs of impaired cognition and executive dysfunction, coupled with difficulty performing multitask, organizing, and planning in daily living; and lastly, stage 4 manifests full-scale dementia. The classification is clinically useful and may serve as a compass to guide a treatment plan, but the size and breadth of sample of case reports are considerably low, thus precluding generalizability of the classification.

To delineate the link between CTE and repetitive mild neurotruama, various questions are pending to be investigated, including antemortem in vivo diagnostic tools, mechanisms of disease, biomarkers, and risk factors such as age and genetics. Recent evidence suggests that in former National Football League players, career longevity and head impact experience during childhood may contribute in earlier onset of cognitive decline[6] and disruption in white matter microstructure, especially in the corpus callosum[7], where interhemispheric communication occurs. Moreover, acute bouts of subconcussive head impact from rugby game negatively altered neuronal functional connectivity in the retrosplenial cortex and posterior cingulate cortex [8], which participate in cognitive and emotional processing. In addition to measuring the neuronal performance using functional magnetic resonance imaging (fMRI), novel cell-based assays coupled with fluorescence resonance energy transfer (FRET) technology to assess tau levels in the brain and blood are currently under development. The FRET can detect an initial step in tau aggregation leading to neurofibrillary tangle formation commonly observed in neurodegenerative disease[9].

Our recent clinical studies suggest that some of neurological system may be impaired and reflective of frequency and magnitude of subconcussive head impact sustained. For example, 10 bouts of soccer headers with an average magnitude of $14.5 \mathrm{~g}$ led to a transient dysfunction in vestibular processing, causing significantly larger postural sway while static and walking[10] Similarly, soccer headings acutely blunted the ocular-motor system, particularly near point of convergence, and the impairment persisted even after 24 hours of resting[11] pointing to the possibility that vulnerability and slow recovery nature of the ocular-motor system. A follow-up prospective longitudinal study in collegiate football players confirmed our finding that the degree of impairment in the ocular-motor function was dependent on the frequency and magnitude of subconcussive head impact sustained[12]. Taken together, although a direct causality between subconcussive head impact exposure and development of CTE remains elusive, it is imperative to employ prospective cohort approaches, rather than case-reports, as to delineate chronic effects of head impact and different factors (e.g., age, gender, genetics) contributing to the disease.

Despite rapid technological advancement, to further understand CTE a constructive and multi-disciplinary effort is a key. First, researchers and media must be alert for and estimate the possible effect of sample bias, discouraging an overstatement from inconclusive observations. Second, establishing age- and gender-dependent "healthy" baseline in various compelling measurements (e.g., functional neural activity[8], sensory systems[3,6] blood biomarkers[13] is warranted. Third, researchers must collaborate with clinical health professionals to longitudinally monitor athletes and military soldiers who may be prone to sustain head impacts. It is crucial to evaluate feasibility and priority of each assessment in the clinical setting. Forth, researchers should consider adopting preclinical randomized clinical multicenter trial protocols, ${ }^{14}$ which have been demonstrated to be a feasible tool to delineate the underlying process of the disease and possibly the effect of treatment. These approaches are needed to inform decision making before researchers embark on large-scale randomized trials of CTE in the future. 
Citation: Lee JH, Kawata K. Protect the player, protect the game: subconcussion and chronic traumatic encephalopathy. J Neurol Neurorehabil Res. 2016;1(2):1-2.

\section{Reference}

1. McKee AC, Stern RA, Nowinski CJ, et al. The spectrum of disease in chronic traumatic encephalopathy. Brain : a journal of neurology. 2013;136(1):43-64.

2. Gardner A, Iverson GL, McCrory P. Chronic traumatic encephalopathy in sport: a systematic review. Br J Sports Med. 2014;48(2):84-90.

3. McKee AC, Cantu RC, Nowinski CJ, et al. Chronic traumatic encephalopathy in athletes: progressive tauopathy after repetitive head injury. J Neuropathol Exp Neurol. 2009;68(7):709-35.

4. Omalu B, Hammers JL, Bailes J, et al. Chronic traumatic encephalopathy in an Iraqi war veteran with posttraumatic stress disorder who committed suicide. Neurosurgical focus. 2011;31(5):E3.

5. Baugh CM, Stamm JM, Riley DO, et al. Chronic traumatic encephalopathy: neurodegeneration following repetitive concussive and subconcussive brain trauma. Brain imaging and behavior. 2012;6(2):244-54.

6. Stamm JM, Bourlas AP, Baugh CM, et al. Age of first exposure to football and later-life cognitive impairment in former NFL players. Neurology. 2015;84(11):1114-20

7. Stamm JM, Koerte IK, Muehlmann M, et al. Age at First Exposure to Football Is Associated with Altered Corpus Callosum White Matter Microstructure in Former
Professional Football Players. Journal of neurotrauma. 2015;32(22):1768-76.

8. Johnson., Neuberger T, Gay M, Hallett M, Slobounov S. Effects of subconcussive head trauma on the default mode network of the brain. Journal of neurotrauma. 2014;31(23):1907-13.

9. Cook CN, Murray ME, Petrucelli L. Understanding biomarkers of neurodegeneration: Novel approaches to detecting tau pathology. Nature medicine. Mar 2015;21(3):219-220.

10. Hwang S, Ma L, Kawata K, et al. Vestibular Dysfunction following Sub-Concussive Head Impact. Journal of neurotrauma. 2016.

11. Kawata K, Tierney R, Phillips J, et al. Effect of Repetitive Sub-concussive Head Impacts on Ocular Near Point of Convergence. International journal of sports medicine. 2016;37(5):405-10.

12. Kawata K, Rubin LH, Lee J, et al. Effect of football subconcussive head impact on ocular near point of convergence. JAMA Ophthalmology. 2016;134(7):763-69.

13. Kawata K, Liu CY, Merkel SF, et al. Blood Biomarkers for Brain Injury: What Are We Measuring? Neuroscience and biobehavioral reviews. 2016; 68:46-73

14. Multi-PART. Multicentre Preclinical Animal Research Team. 2014; www.den.ed.ac.uk/multipart/. June 20, 2016.

\section{*Correspondence to:}

Keisuke Kawata

Indiana University-Bloomington, Department of Kinesiology

Bloomington

E-mail: kkawata@indiana.edu

Tel: 8128566035 